\title{
A New Unified Electro-Gravity Theory for the Electron
}

\author{
Nirod K. Das \\ Department of Electrical and Computer Engineering, Tandon School of Engineering, \\ New York University, 5 Metrotech Center, Brooklyn, NY 11201
}

(Dated: August 13, 2019)

\begin{abstract}
A rigorous model for the electron is presented by generalizing the Coulomb's Law or Gauss's Law of electrostatics, using a unified theory of electricity and gravity. The permittivity of the free-space is allowed to be variable, dependent on the energy density associated with the electric field at a given location, employing generalized concepts of gravity and mass/energy density. The electric field becomes a non-linear function of the source charge, where concept of the energy density needs to be properly defined. Stable solutions are derived for a spherically symmetric, surface-charge distribution of an elementary charge. This is implemented by assuming that the gravitational field and its equivalent permittivity function is proportional to the energy density, as a simple firstorder approximation, with the constant of proportionality referred to as the Unifield Electro-Gravity (UEG) constant. The stable solution with the lowest mass/energy is assumed to represent a "static" electron without any spin. Further, assuming that the mass/energy of a static electron is half of the total mass/energy of an electron including its spin contribution, the required UEG constant is estimated. More fundamentally, the lowest stable mass of a static elementary charged particle, its associated classical radius, and the UEG constant are related to each other by a dimensionless constant, independent of any specific value of the charge or mass of the particle. This dimensionless constant is numerologically found to be closely related to the the fine structure constant. This possible origin of the fine structure constant is further strengthened by applying the proposed theory to successfully model the Casimir effect, from which approximately the same above relationship between the UEG constant, electron's mass and classical radius, and the fine structure constant, emerges.
\end{abstract}

\section{INTRODUCTION}

The electron is the most fundamental charged particle of nature [1], carrying the smallest mass among all known charged particles, and is classified as a lepton in the standard model of particle physics [2, 3]. It plays a fundamental role in our everyday nature as a basic building block of all chemical elements, which consist of one or more electrons orbiting in different spatial forms around an oppositely charged, massive central nucleus [4, 5]. Different physical parameters of the electron - its charge, mass, as well as the spin angular momentum and the magnetic moment [6]- have been measured in great precision. The electron's characteristics in an electromagnetic field have also been successfully modeled using quantum mechanical wave functions 9 11] and quantum electro-dynamics [12]. However, any internal structure of the electron, and the origin of its mass, remain mysterious. It is sometimes considered to be a "point particle" with no particular internal structure 13 . However, the electromagnetic energy, or its equivalent mass, for the point-particle would be infinite [14, which is unphysical and inconsistent with the finite measured mass of the electron [6]. Further, the question of how the electronic charge could withstand the repulsive force due to its own electric field [14, which is infinite for the point-structure with a zero radius (or even a finite value if the electron had a non-zero radius), can not be properly answered.

In this paper we model an electron using a proposed new theory, referred to as a Unified Electro-Gravity (UEG) theory. The theory attempts to unify the concept of the electric field surrounding a source charge, as de- fined by the Coulomb's Law or Gauss' Law of electrostatics [15 17], together with a generalized concept of gravity produced due to energy density associated with the electric field, that would be consistent with the Newton's Law of Gravity [18, 19. The permittivity of the "free-space" around a charge, which is conventionally assumed to be a fixed constant in the Coulomb's Law or Gauss' Law, is now modeled as a functional distribution, dependent on the distribution of the electric field or its associated energy density. The permittivity function needs to be consistent with the Newton's Law of gravity, where a gravitational field is recognized to be directly proportional to the gradient of the inverse-permittivity function. Accordingly, such an "unified electo-gravitational (UEG)" field may be modeled as a non-linear field, where the permittivity distribution is a general function of the source charge, or equivalently the electric field is a non-linear function of the source charge. Under this non-linear condition, the definition of energy density and its expression in terms of the source charge or the electric field, used in conventional electromagnetic theory, may have to be properly modified.

With a proper definition of the energy density associated with the non-linear UEG field, and a suitable relationship between the gravitational field and the energy density, the permittivity function surrounding a spherically symmetric surface-charge distribution may be solved, either analytically or numerically. Consequently, the total energy, or its equivalent mass as per special relativity, may be derived as a function of the charge radius. It is discovered that stable solutions, where the first derivative of the total energy with respect to the charge 
radius is zero, and the second derivative positive, are possible for certain discrete values of the charge radius. The derivation assumes a simple proportional relationship between the energy density and the UEG field, with the constant of proportionality referred to as the UEG constant. It maybe reasonable to assume that the stable solution having the smallest possible mass/energy is associated with the mass/energy of an ideal "static electron" that does not spin around itself. Further, the mass of the static electron may be ideally assumed to be half of the total mass of an electron that includes its spin contribution. Accordingly, by reverse deduction, the UEG constant can be calculated, and is recognized as a new fundamental constant of nature. This is a significant fundamental development.

The new UEG constant is defined as the gravitational acceleration per unit energy density, carrying a dimension of $\left(\mathrm{m} / \mathrm{s}^{2}\right) /\left(\mathrm{J} / \mathrm{m}^{3}\right)$. More significantly, a dimensionless constant relating the UEG constant, the stable static mass, and its associated classical radius, is identified which would apply to any basic charge particle, independent of the specific charge or mass of the particle. The value of this dimensionless constant is numerologically recognized to be closely related to the fine structure constant 20]. This general finding may suggest a much broader scope of application of the UEG theory to other known elementary particles in the standard model of particle physics [2, 3, 21, 22, which might be associated with different effective values of the UEG constant, resulting in different mass and classical radii of the particles, while they carry the same value of the elementary charge as the electron. Considering the broad reach of the fine structure constant in quantum mechanics and electro-dynamics [20, 23, 24, the recognition that the fine-structure constant may have its fundamental origin in the UEG theory may carry profound theoretical and fundamental implications.

The possible origin of the fine structure constant, as suggested above, is further strengthened by applying the proposed UEG theory to model the Casimir effect [25, 26]. The Casimir attractive force between two parallel conducting plates is modeled as a UEG effect due to any non-zero electric field that might escape out of a conductor through any spatial gaps between spherical electrons on the conductor's surface, which are periodically distributed in the form of square unit cells. Equating this force with the expected Casimir force as understood based on quantum mechanics and vacuum zero-point energy [25, 27], approximately the same above relationship between the UEG constant, electron's mass and classical radius, and the fine structure constant, emerges. This would strongly suggest that the UEG theory is the origin of both the Casimir effect, as well as the fine-structure constant.

\section{GRAVITY AS GRADIENT OF FREE-SPACE PERMITTIVITY}

A massive body in a gravitational field $\bar{E}_{g}$ experiences a force $\bar{F}$ in a certain direction in space. In the theory of general relativity this force is seen as a result of curvature of the surrounding "free-space" [28]. The force may be alternatively modeled by considering the permittivity $\epsilon$ of the surrounding "free-space" to be a non-uniform function $\epsilon(\bar{r})$ of the location $\bar{r}$ (unlike a constant value $\epsilon=\epsilon_{0}$ normally used), and assuming that the mass of a given body at a particular location is a function of the local permittivity (see Fig.1). As the mass is displaced from one location over an incremental distance along a given direction, its mass or equivalent energy is also incrementally changed due to the incremental change in the permittivity associated with the displacement. This change in energy per unit displacement in the given direction would be equal to the force component in the particular direction. Accordingly, the gravitational field is modeled in terms of gradient of the permittivity function of the "free-space" medium.

We assume that the mass $m$ or the equivalent energy $W=m c^{2}$, where $c$ is the speed of light in an isolated free-space, is inversely proportional to the permittivity $\epsilon$, or directly proportional to $\underline{\epsilon}=1 / \epsilon$. This is in consistency with the energy $W=\frac{q^{2}}{8 \pi \epsilon r_{q}}$ of a spherical surface charge $q$ of radius $r_{q}$, placed in a medium with permittivity $\epsilon$.

$$
\begin{gathered}
\underline{\epsilon}(\bar{r})=\underline{\epsilon}_{0} \underline{\epsilon}_{r}(\bar{r}), \epsilon=\epsilon_{0} \epsilon_{r}, \underline{\epsilon}=\frac{1}{\epsilon}=\frac{1}{\epsilon_{0} \epsilon r}=\underline{\epsilon}_{0} \underline{\epsilon}_{r}, \\
\underline{\epsilon}_{0}=\frac{1}{\epsilon_{0}}, \underline{\epsilon}_{r}=\frac{1}{\epsilon_{r}} ; \\
m(\bar{r}) \alpha \underline{\epsilon}(\bar{r}), m(\bar{r})=m_{0} \underline{\epsilon}_{r}(\bar{r}), \\
m_{0}=m\left(\underline{\epsilon} \rightarrow \underline{\epsilon}_{0}, \underline{\epsilon}_{r} \rightarrow 1\right), \underline{\epsilon}_{r}=\frac{1}{\epsilon_{r}}=\frac{\underline{\epsilon}}{\underline{\epsilon}_{0}}=\frac{m}{m_{0}} ; \\
\bar{E}_{g}=\frac{\bar{F}}{m_{0}}=\frac{-\bar{\nabla} W(\bar{r})}{m_{0}}=\frac{-\bar{\nabla}\left[m(\bar{r}) c^{2}\right]}{m_{0}} \\
=\frac{-\bar{\nabla}\left[m_{0} \underline{\epsilon}_{r}(\bar{r}) c^{2}\right]}{m_{0}}=-c^{2} \bar{\nabla}_{\epsilon_{r}}(\bar{r}) .
\end{gathered}
$$

\section{A. Gravitational Field and Permittivity Function in a Region with Energy/Mass Distribution}

Consider the gravitational field produced by a body of mass of $m_{0}$, as per the Newton's Law of Gravitation, exerting a force on an external mass $\delta m_{0}$. The permittivity function around the mass $m_{0}$ may be expressed using the model (1) developed above.

$$
\begin{gathered}
\bar{F}=-\frac{G m_{0} \delta m_{0}}{r^{2}} \hat{r}, \\
\bar{E}_{g}=\frac{\bar{F}}{\delta m_{0}}=-\frac{G m_{0}}{r^{2}} \hat{r}=-c^{2} \bar{\nabla}_{\epsilon_{r}}(\bar{r})=-c^{2} \frac{\partial \underline{\epsilon}_{r}(r)}{\partial r} \hat{r}, \\
\frac{\partial \underline{\epsilon}_{r}(r)}{\partial r}=\frac{G m_{0}}{c^{2} r^{2}}, \underline{\epsilon}_{r}=1-\frac{G m_{0}}{c^{2} r}, \underline{\epsilon}_{r}(r \rightarrow \infty)=1 .
\end{gathered}
$$




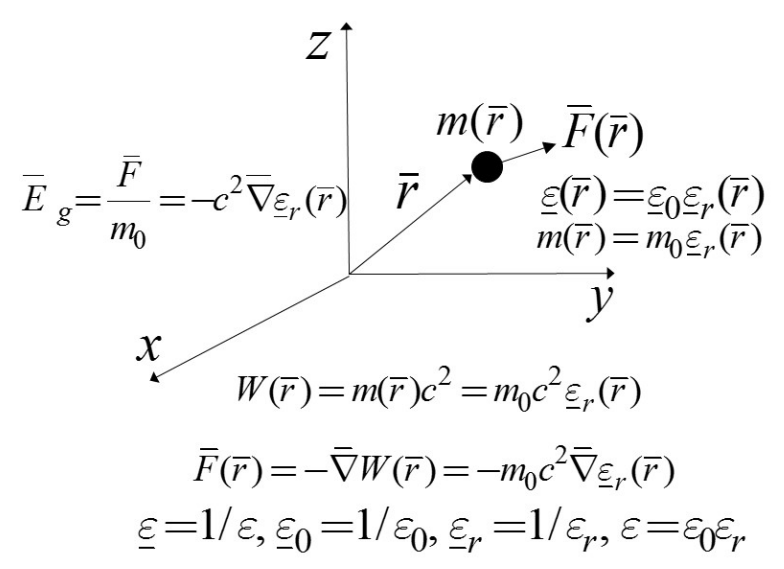

FIG. 1.

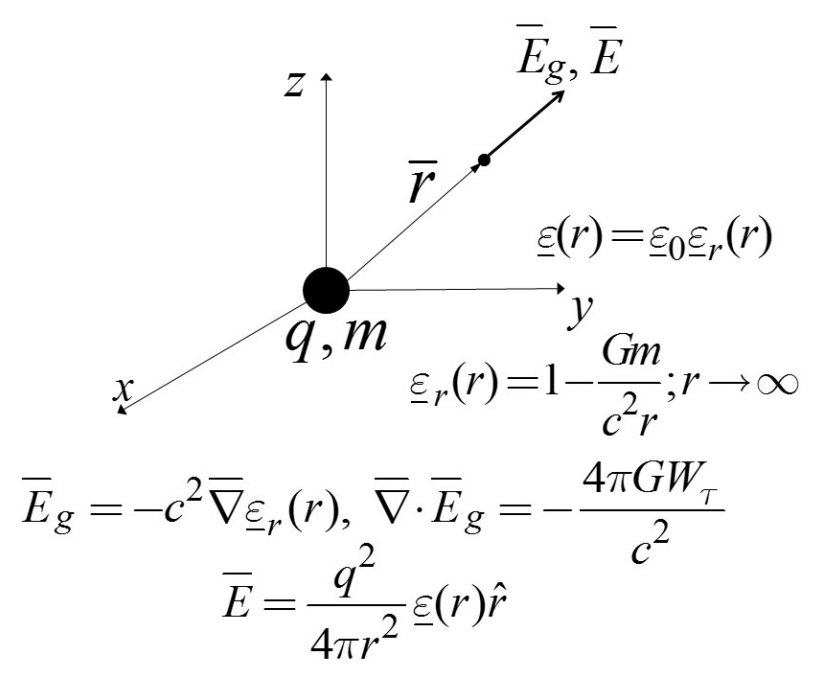

FIG. 2 .

The above result would be applicable for all distances $r>0$ for an ideal point-body, and would apply only outside the body for a body of non-zero radius.

The permittivity function for a body with distributed mass/energy, such as an electric charge, may be similarly developed (see Fig 2), by relating the divergence of the gravitational field $\bar{E}_{g}$ in 2 to the mass-density $m_{\tau 0}$. The mass-density $m_{\tau 0}$ of a distributed body at a particular location is defined as the mass per a unit elemental volume $d \tau=1$ at the given location. The equivalent energy-density $W_{\tau 0}=c^{2} m_{\tau 0}$.

$$
\begin{gathered}
\bar{\nabla} \cdot \bar{E}_{g}=-4 \pi G m_{\tau 0}=\bar{\nabla} \cdot\left(-c^{2} \bar{\nabla}_{\epsilon_{r}}\right), \\
\bar{\nabla} \cdot \bar{\nabla} \underline{\epsilon}_{r}=\frac{4 \pi G m_{\tau 0}}{c^{2}}=\frac{4 \pi G W_{\tau 0}}{c^{4}}
\end{gathered}
$$

\section{MODELING ENERGY DENSITY IN A NON-LINEAR MEDIUM AROUND AN ELECTRIC CHARGE}

In the unified electro-gravity (UEG) model, the permittivity distribution of the free-space is dependent upon the energy density distribution, which is dependent upon the source charge. This is unlike a linear dielectric medium where the permittivity function is independent of the field strength or the source charge. Having the permittivity distribution to be a function of the source charge, is equivalent to having the electric field distribution to be a non-linear function of the source charge. The energy density in such a non-linear medium needs to be properly modeled, starting from the fundamentals. This would result in a general expression for the energy density for a non-linear medium, which may be verified with a standard expression of the energy density for a linear medium, as a special case when the permittivity is a constant independent of the charge.

The electric field $\bar{E}$ and the electric flux density $\bar{D}$ produced due to a charge $q$, at a distance $r$ from the center of the charge, in the presence of a permittivity distribution $\epsilon(r)=1 / \underline{\epsilon}(r)$ may be expressed using the Coulomb's Law.

$$
\bar{E}=\frac{q}{4 \pi r^{2} \epsilon(r)} \hat{r}, \bar{D}=\frac{q}{4 \pi r^{2}} \hat{r}, \bar{E}=\frac{\bar{D}}{\epsilon(r)}=\underline{\epsilon}(r) \bar{D} .
$$

Let us calculate an incremental energy $d W$ required in moving an incremental charge $d q$ from infinity to a radius $r=r_{q}$, using the above electric field. This is equivalent to having $d W=V(q) d q$ using a potential concept, where $V(q)$ is the potential (function of $q$ ) at the radius $r=r_{q}$. Integrating the $d W$ over the total charge $q$ would give the total energy $W$.

$$
\begin{gathered}
d W=d q \int_{r_{q}}^{\infty} \bar{E}(q) \cdot \overline{d r} \\
=V\left(q ; r=r_{q}\right) d q=\iiint_{\tau ; r>r_{q}} d W_{\tau} d \tau, \\
W=\int_{q=0}^{q} d W=\iiint_{\tau ; r>r_{q}}\left(\int_{q=0}^{q} d W_{\tau}\right) d \tau .
\end{gathered}
$$

The incremental charge $d q$ may be expressed in terms of an incremental change in the electric flux density $d \bar{D}$ using Gauss Law. The incremental energy $d W$ can then be expressed as an integral over the external volume $\tau ; r>r_{q}$ using the divergence theorem. 


$$
\begin{gathered}
d W=V\left(q ; r=r_{q}\right) d q=\iint_{S, r=r_{q}+\delta} V(q) d \bar{D} \cdot \overline{d s} \\
=\iiint_{\tau ; r>r_{q}} \bar{\nabla} \cdot(-V(q) d \bar{D}) d \tau \\
=\left[\iiint_{\tau ; r>r_{q}}(-\bar{\nabla} V(q) \cdot d \bar{D}) d \tau\right. \\
\left.+\iiint_{\tau ; r>r_{q}}-V(q)(\bar{\nabla} \cdot d \bar{D}) d \tau\right] \\
=\iiint_{\tau ; r>r_{q}} \bar{E}(q) \cdot d \bar{D} d \tau \\
=\iiint_{\tau ; r>r_{q}} \underline{\epsilon}(q) \bar{D}(q) \cdot d \bar{D} d \tau \\
=\iiint_{\tau ; r>r_{q}} \frac{\epsilon}{(q)} \frac{1}{2} \frac{\partial}{\partial q}(\bar{D}(q) \cdot \bar{D}(q)) d q d \tau \\
=\iiint_{\tau ; r>r_{q}} \frac{1}{2} \underline{\epsilon}(q) \frac{\partial|\bar{D}|^{2}}{\partial q} d q d \tau=\iiint_{\tau ; r>r_{q}} d W_{\tau} d \tau \\
\bar{\nabla} \cdot d \bar{D}=0 \text { in } \tau .
\end{gathered}
$$

We have now established an expression for an incremental energy density $d W_{\tau}$, which may be integrated over the total charge $q$ to obtain the required expression of the energy density $W_{\tau}$. The general expression may be verified to be the conventional energy density for a linear medium, when the permittivity is a constant independent of the charge $q$. The total energy $W$ can then be calculated as the volume integral of the energy density $W_{\tau}$.

$$
\begin{aligned}
d W_{\tau} & =\frac{1}{2} \underline{\epsilon}(q) \frac{\partial|\bar{D}|^{2}}{\partial q} d q \\
W=\iiint_{\tau} W_{\tau} d \tau & =\iiint_{\tau}\left(\int_{q=0}^{q} d W_{\tau}\right) d \tau=m_{0} c^{2}
\end{aligned}
$$

In equivalency to a conventional definition of the energy density for a linear medium, it may be useful to define a new variable $\underline{\epsilon}^{\prime}$ for a non-linear medium. The conventional expression of the energy density for a linear medium, with the inverse-permittivity $\epsilon$ for the linear medium simply substituted by the new equivalent variable $\underline{\epsilon}^{\prime}$, would be valid as well for the non-linear medium.

$$
\begin{gathered}
W_{\tau}=\int_{q=0}^{q} d W_{\tau}=\int_{q=0}^{q} \frac{1}{2} \underline{\epsilon}(q) \frac{\partial|\bar{D}|^{2}}{\partial q} d q=\frac{1}{2} \underline{\epsilon}^{\prime}|\bar{D}|^{2}=\frac{1}{2} \underline{\epsilon}_{0} \underline{\epsilon}_{r}^{\prime}|\bar{D}|^{2} \\
\underline{\epsilon}^{\prime}=\frac{1}{|\bar{D}|^{2}} \int_{q=0}^{q} \underline{\epsilon}(q) \frac{\partial|\bar{D}|^{2}}{\partial q} d q=\frac{1}{q^{2}} \int_{q=0}^{q} \underline{\epsilon}(q) \frac{\partial q^{2}}{\partial q} d q \\
=\frac{2}{q^{2}} \int_{q=0}^{q} \underline{\epsilon}(q) q d q=\frac{2 \epsilon_{0}}{q^{2}} \int_{q=0}^{q} \underline{\epsilon}_{r}(q) q d q=\underline{\epsilon}_{0} \underline{\epsilon}_{r}^{\prime} .
\end{gathered}
$$

\section{A UNIFIED ELECTRO-GRAVITY MODEL FOR AN ELEMENTARY CHARGE, WITH A NEW DEFINITION OF THE ENERGY DENSITY}

For a given total energy $W$, the energy density $W_{\tau}$ we derived may not be unique. An alternate expression of the energy density $W_{\tau}^{\prime}$ may be defined by adding a distribution $f$ to the original energy density $W_{\tau}$, such that the $W_{\tau}^{\prime}$ would result in the same total energy $W$ when integrated over the total volume $\tau$ as that due to the original energy density $W_{\tau}$. Accordingly, a fixed total energy $W$ is redistributed into the different energy densities $W_{\tau}$ and $W_{\tau}^{\prime}$ inside the volume $\tau$. This can be accomplished by having the additional distribution $f$ expressed as divergence of a suitable vector distribution $\bar{U}$, which is identically zero everywhere outside the volume $\tau$.

$$
\begin{gathered}
W=\iiint_{\tau} W_{\tau} d \tau=m_{0} c^{2}, \\
W_{\tau}=\frac{\Delta W}{\triangle \tau}=\frac{1}{2} \epsilon^{\prime}|\bar{D}|^{2}=\frac{1}{16 \pi^{2} r^{4} \epsilon_{0}} \int_{0}^{q} q \underline{\epsilon}_{r}(q) d q, \\
W_{\tau}^{\prime}=W_{\tau}+f, \\
f=\bar{\nabla} \cdot \bar{U}=\bar{\nabla} \cdot(U \hat{u}) ; \bar{U}=0 \text { outside of } \tau, \\
=\int=\iiint_{\tau} W_{\tau}^{\prime} d \tau \\
=\iiint_{\tau}\left(W_{\tau}+f\right) d \tau=\iiint_{\tau}\left(W_{\tau}+\bar{\nabla} \cdot \bar{U}\right) d \tau \\
=\iiint_{\tau} W_{\tau} d \tau+\iiint_{\tau} \bar{\nabla} \cdot \bar{U} d \tau \\
=\iiint_{\tau} W_{\tau} d \tau+\iint_{S} \bar{U} \cdot \overline{d s}=\iiint_{\tau} W_{\tau} d \tau,
\end{gathered}
$$

$$
\begin{gathered}
U\left(W_{\tau}\right): U\left(W_{\tau}=0\right)=0, \hat{u}=\hat{r} ; \\
W_{\tau}=0 \text { outside of } \tau, \bar{U}=\zeta W_{\tau} \hat{r} .
\end{gathered}
$$

An alternate expression of the energy density $W_{\tau}^{\prime}$, as in (9), would require revision of the Poynting theorem of the electromagnetic theory [29, 30], in order to re-establish proper relationship between different energy and power associated with an electromagnetic field.

Theoretically, there are many possible expressions for the vector function $\bar{U}$. A simple, physically meaningful proposition is to express the function $\bar{U}$ 11, referred to as the UEG function, proportional to the original energy density $W_{\tau}$, and directed toward the center of mass/gravity of the particle.

Consider the external free-space region of a "neutral" material body, that appears to be charge-less to an external observer, with the electromagnetic field and its associated energy density in the external region equal to zero. With the above choice of the UEG function $\bar{U}$ (11), no new, special treatment would be required to model the gravitational field in the external region, because the original as well as the revised energy densities of (9), $W_{\tau}$ and $W_{\tau}^{\prime}$ respectively, would be zero in this region. Further, with the choice of the UEG function (11), the total energy $W$, or its equivalent mass $m=W / c^{2}$ of the neutral body, as seen by an external observer, would remain the same whether the $W$ is calculated by integrating the original or the revised energy density in the internal region, as per the deduction in (10). Accordingly, Newtonian gravitational field in the external region of such neutral material bodies would remain unaffected by the 
new UEG theory, which would be consistent with observation.

The selected UEG function $\bar{U}$ 11 could be non-zero in the internal region of a neutral body discussed above, due to non-zero electromagnetic fields associated with any charged sub-structure internal to the body. This would lead to having the revised energy density $W_{\tau}^{\prime}$ in (9) to be different from the original energy density $W_{\tau}$ in the internal region. Accordingly, it would require a revised treatment for modeling the gravitational field, in the internal charged region of such a neutral material body, or for that matter in any general region in the presence of a non-zero electromagnetic field.

The new alternate expression for the energy density $W_{\tau}^{\prime}$ of $(9)$, using the new UEG function $\bar{U}$ of (11), may now be substituted for the original energy density $W_{\tau 0}=W_{\tau}$ in the UEG modeling of the gravitational field in (3).

$$
\begin{gathered}
\bar{\nabla} \cdot \bar{E}_{g}=-c^{2} \bar{\nabla} \cdot \bar{\nabla} \underline{\epsilon}_{r}=-4 \pi G m_{\tau}^{\prime}=-\frac{4 \pi G W_{\tau}^{\prime}}{c^{2}} \\
=-\frac{4 \pi G}{c^{2}}\left(W_{\tau}+\bar{\nabla} \cdot \bar{U}\right)=-\frac{4 \pi G}{c^{2}} W_{\tau}-\bar{\nabla} \cdot\left(\gamma W_{\tau} \hat{r}\right), \\
\frac{4 \pi G}{c^{2}} \bar{U}=\frac{4 \pi G}{c^{2}} \zeta W_{\tau} \hat{r}=\gamma W_{\tau} \hat{r} .
\end{gathered}
$$

It may be observed from the above expression of the gravitational field $\bar{E}_{g}$, that the new UEG function $\bar{U}$, which was introduced for an alternate definition of the energy density $W_{\tau}^{\prime}$ in (11), would be equivalent to having an additional gravitational field equal to $-\gamma \bar{U}$, referred to as the UEG field. The parameter $\gamma$ in 12 is a new scalar constant, referred to as the UEG constant, which is related to the constant $\zeta$ used in (11).

\section{A. Series Solution for $\underline{\epsilon}_{r}$, with a Strong UEG Force Assumption}

We will solve for the inverse-relative permittivity function $\underline{\epsilon}_{r}(r)$, by expanding it as power-series of $r^{-i}$ with unknown coefficients $b_{i}$, and then solve for the coefficients in order to satisfy the above UEG relation (12). In the limit of large distance $r$, the $\underline{\epsilon}_{r}(r)$ needs to satisfy the Newtonian gravitational field (2) due to the particle mass $m_{0}$, approaching unity at infinite distance $r \rightarrow \infty$. The limiting conditions would fix the first two coefficients $b_{0}$ and $b_{1}$.

$$
\underline{\epsilon}_{r}(r, q)=\sum_{i=0}^{\infty} b_{i} r^{-i}, b_{0}=1, b_{1}=-\frac{G m_{0}}{c^{2}} .
$$

This assumes that the surrounding medium at infinite distance from the particle is a free-space with $\epsilon=\epsilon_{0}$, $\epsilon_{r}=1=1 / \epsilon_{r}=\underline{\epsilon}_{r}$, and the $m=m_{0}$ is the mass of the particle when measured in the free-space medium. If the surrounding medium is different from the free-space, with $\epsilon=\epsilon_{r} \epsilon_{0}, 1 / \epsilon_{r}=\underline{\epsilon}_{r} \neq 1$, then the above solution 13 needs to be scaled with $b_{0}=\underline{\epsilon}_{r}$ and $b_{1}=\frac{G m_{0} \underline{\epsilon}_{r}}{c^{2}}$. It may be shown from the following iterative solution for the $\underline{\epsilon}_{r}(r)$, that each term in the series expression of 113 , and therefore the entire expression of $(13)$, would be multiplied by the $\underline{\epsilon}_{r}(r \rightarrow \infty)$ of the surrounding medium, in order to obtain the $\underline{\epsilon}_{r}(r)$ for the particle in the given surrounding medium. Further, the mass function $m(r)$ for the particle measured in the given surrounding medium, as derived in section IVB using the above scaled $\underline{\epsilon}_{r}$, may be shown to be equal to $m=m_{0} \underline{\epsilon}_{r}(r \rightarrow \infty)$, as expected in section II. For simplicity, in the following derivations we will assume the surrounding medium to be free-space, the results from which may be properly scaled as needed for any other surrounding medium.

We may assume that the new UEG field $\gamma \bar{U}$ is much stronger than the conventional Newtonian gravitational field of the charge particle, contributed due to the original energy density $W_{\tau}$. This is because the conventional Newtonian gravitational field of an elementary charge is known to be very week, having a negligible (essentially no) effect on the permittivity function. It may be shown, that this assumption would be valid given the radius $r$ of the charge particle is much larger than the radius $r_{0}$ of a black-hole produced by an elementary charge $q$, with a mass equal to the classical mass $q^{2} /\left(8 \pi \epsilon_{0} r_{0} c^{2}\right)$ of the charge with the radius $r_{0}$.

$$
\begin{gathered}
\bar{\nabla} \cdot \bar{E}_{g}=-c^{2} \bar{\nabla} \cdot \bar{\nabla} \underline{\epsilon}_{r} \simeq-\bar{\nabla} \cdot\left(\gamma W_{\tau} \hat{r}\right) \\
=-\bar{\nabla} \cdot\left(\frac{\gamma \hat{r}}{16 \pi^{2} r^{4} \epsilon_{0}} \int_{0}^{q} q \underline{\epsilon}_{r}(q, r) d q\right) \\
=-\bar{\nabla} \cdot\left(\frac{\gamma q^{2} \epsilon_{r}^{\prime}(r) \hat{r}}{32 \pi^{2} r^{4} \epsilon_{0}}\right), r>>r_{0}=\sqrt{\frac{G q^{2}}{8 \pi \epsilon_{0} c^{4}}}, \\
\bar{E}_{g} \simeq-\gamma W_{\tau} \hat{r}=-\frac{\gamma \hat{r}}{16 \pi^{2} r^{4} \epsilon_{0}} \int_{0}^{q} q \underline{\epsilon}_{r}(q, r) d q \\
=-\frac{\gamma q^{2} \underline{\epsilon}_{r}^{\prime}(r) \hat{r}}{32 \pi^{2} r^{4} \epsilon_{0}}, r>>r_{0}=\sqrt{\frac{G q^{2}}{8 \pi \epsilon_{0} c^{4}}} .
\end{gathered}
$$

The expression (8) for the energy density $W_{\tau}$ in a nonlinear medium is used in the above derivation. Assuming that the charge distribution and the UEG solution are spherically symmetric, the differential operators in the above expression can be expressed in terms of derivatives with respect to the radius. Substituting the series expression of (13) in (14) we get,

$$
\begin{gathered}
\frac{\partial}{\partial r} \underline{\epsilon}_{r} \simeq \frac{1}{c^{2}}\left(\gamma W_{\tau}\right)=\frac{\gamma}{16 \pi^{2} r^{4} c^{2} \epsilon_{0}} \int_{0}^{q} q \underline{\epsilon}_{r}(r, q) d q \\
=\frac{3 r_{\mu}^{3}}{2 q^{2} r^{4}} \int_{0}^{q} q \underline{\epsilon}_{r}(r, q) d q, r_{\mu}^{3}=\frac{\gamma q^{2}}{24 \pi^{2} c^{2} \epsilon_{0}} \propto q^{2} \\
r_{\mu}=\left(\frac{\gamma q^{2}}{24 \pi^{2} c^{2} \epsilon_{0}}\right)^{1 / 3}=5.14 \times 10^{-16} \gamma^{1 / 3} \\
\frac{\partial^{2} \epsilon_{r}(r, q)}{\partial\left(r^{-3}\right) \partial\left(q^{2}\right)} \simeq-\frac{r_{\mu}^{3}}{4 q^{2}} \underline{\epsilon}_{r}(r, q) .
\end{gathered}
$$




$$
\begin{gathered}
\sum_{i=0}^{\infty} b_{i}(-i) r^{-(i+1)} \simeq \frac{3 r_{\mu}^{3}}{2 q^{2}} \sum_{i=0}^{\infty}\left(\int_{0}^{q} q b_{i}(q) d q\right) r^{-(i+4)}, \\
=\frac{3 r_{\mu}^{3}}{2 q^{2}} \sum_{i=3}^{\infty}\left(\int_{0}^{q} q b_{i-3}(q) d q\right) r^{-(i+1)} .
\end{gathered}
$$

The above relation provides an iterative solution for the coefficients $b_{i}$.

$$
\begin{gathered}
b_{i} \simeq-\frac{3 r_{\mu}^{3}}{2 q^{2}(i)}\left(\int_{0}^{q} q b_{i-3}(q) d q\right), \\
b_{0}=1, \quad b_{i}=0 ; \quad i \neq 3 k=0,3,6,9 \cdots .
\end{gathered}
$$

The series may be re-sequenced with $a_{k}=b_{3 k}$, because all coefficients $b_{i}$ for $i$ other than $i=3 k=0,3,6,9 \cdots$ are zero.

$$
\begin{aligned}
a_{k}= & b_{3 k} \simeq-\frac{r_{\mu}^{3}}{q^{2}(2 k)}\left(\int_{0}^{q} q b_{3 k-3}(q) d q\right) \\
= & -\frac{r_{\mu}^{3}}{q^{2}(2 k)}\left(\int_{0}^{q} q a_{k-1}(q) d q\right) .
\end{aligned}
$$

From the above iterative relation it may be recognized that $a_{k}$ would be proportional to $q^{2 k}$. This condition may be used to simplify the iterative relation for $a_{k}$ and then solve for all the coefficients $a_{k}$ starting with the known coefficient $a_{0}=1$.

$$
\begin{gathered}
a_{k}(q) \propto q^{2 k}, a_{0}=1, a_{1}=-\frac{r_{\mu}^{3}}{2 \times 2}, \\
a_{2}=\frac{r_{\mu}^{6}}{2 \times 2 \times 4 \times 4} ; a_{k}=-a_{k-1} \frac{r_{\mu}^{3}}{(2 k)(2 k)} .
\end{gathered}
$$

The series expression for the inverse-relativepermittivity function $\underline{\epsilon}_{r}(r)$ may be re-formatted as a power series of $t^{2 k}$, where $t$ is a normalized variable $t=\left(r_{\mu} / r\right)^{1.5}$, with corresponding normalized coefficients $a_{k}^{\prime}$.

$$
\begin{gathered}
\underline{\epsilon}_{r}(r)=\sum_{i=0}^{\infty} b_{i} r^{-i} \simeq \sum_{k=0}^{\infty} a_{k} r^{-3 k} \\
=\sum_{k=0}^{\infty} a_{k}^{\prime}\left(\frac{r \mu}{r}\right)^{3 k}=\sum_{k=0}^{\infty} a_{k}^{\prime} t^{2 k} \\
a_{k}^{\prime} r_{\mu}^{3 k}=a_{k}, \quad t=\left(\frac{r \mu}{r}\right)^{1.5}, a_{k}^{\prime}=-a_{k-1}^{\prime} \frac{1}{(2 k)(2 k)}, \\
a_{k}^{\prime}=\frac{(-1)^{k}}{2^{2 k}(k !)^{2}}, \quad k !=(k)(k-1)(k-2) \cdots(1), \\
\underline{\epsilon}_{r}(r) \simeq 1-\frac{t^{2}}{2^{2}[1 !]^{2}}+\frac{t^{4}}{2^{4}[2 !]^{2}}-\frac{t^{6}}{2^{6}[3 !]^{2}}+\cdots \\
=J_{0}(t)
\end{gathered}
$$

The above series is recognized as the zeroth-order Bessel function $J_{0}(t)$ 31]. The corresponding effective function $\epsilon_{r}^{\prime}=1 / \epsilon_{r}^{\prime}$ may be deduced from 22 using the definition (8), and is similarly recognized in relation to the firstorder Bessel function $J_{1}(t)$ 31.

$$
\begin{aligned}
\underline{\epsilon}_{r}^{\prime}(r) & =\frac{2}{q^{2}} \int_{0}^{q} q \underline{\epsilon}_{r}(q, r) d q \\
\simeq 1-\frac{t^{2}}{2^{2}[1 !]^{2} \times 2} & +\frac{t^{4}}{2^{4}[2 !]^{2} \times 3}-\frac{t^{6}}{2^{6}[3 !]^{2} \times 4}+\cdots \\
& =(2 / t) J_{1}(t),
\end{aligned}
$$

The inverse-relative permittivity function $\underline{\epsilon}_{r}=1 / \epsilon_{r}$ of 222), as well as the corresponding effective function $\underline{\epsilon}_{r}^{\prime}=$ $1 / \epsilon_{r}$ of 23 are plotted in Fig 3 as a function of the normalized radius $r_{\mu} / r=t^{2 / 3}$.

The function $\underline{\epsilon}_{r}=1 / \epsilon_{r}$ that would have resulted if a conventional energy density for a linear medium (see (14]8) were used (incorrectly) in the above derivation of section IV A where the effective function $\underline{\epsilon}_{r}^{\prime}=1 / \epsilon_{r}^{\prime}$ from (8) that defines the energy density would be equal to the function $\epsilon_{r}=1 / \epsilon_{r}$, is expressed in (24), and is also plotted in Fig 3 for reference.

$$
\begin{aligned}
\underline{\epsilon}_{r}(r)=\underline{\epsilon}_{r}^{\prime}(r) \simeq 1 & -\frac{t^{2}}{2^{2} 1 !}+\frac{t^{4}}{2^{4} 2 !}-\frac{t^{6}}{2^{6} 3 !}+\cdots \\
& =e^{-t^{2} / 2} .
\end{aligned}
$$

Notice in the Fig 3 that the function $\underline{\epsilon}_{r}$ of 22 (and the corresponding effective function $\underline{\epsilon}_{r}^{\prime}$ of 23 ), derived using the rigorous definition of the energy density (8) for a non-linear medium, exhibits an oscillatory behavior changing its sign from positive to negative values and vice versa. This is in contrast with the result for $\underline{\epsilon}_{r}=1 / \epsilon_{r}=$ $\underline{\epsilon}_{r}^{\prime}=1 / \epsilon_{r}^{\prime}$ from (24) (using a simplistic (incorrect) UEG model), which monotonically approaches zero with no oscillatory behavior. The rigorously derived, oscillatory behavior of the $\underline{\epsilon}_{r}=1 / \epsilon_{r}$ and $\underline{\epsilon}_{r}^{\prime}=1 / \epsilon_{r}^{\prime}$ functions is a key development, which would lead also to an oscillatory behavior of the total energy/mass of the charge particle as a function of radius, to be established in the following section. This would allow the charge particle to maintain a stable structure at discrete values of radius, where the total energy/mass of the particle would be locally minimum.

From Fig 3, it may be noted that at discrete locations where $\underline{\epsilon}_{r}$ of (22) is zero, the corresponding $\underline{\epsilon}_{r}^{\prime}$ of 23 is non-zero, and vice versa. Accordingly, the energy density $W_{\tau}$ of (8) would be non-zero, at the discrete locations where the field $\bar{E}$ of $(4)$ is zero, and vice versa. This is unlike a conventional field in a "free-space" medium having a fixed relative permittivity $\epsilon_{r}=1$, in which case a non-zero or zero electric field is respectively associated with a non-zero or zero energy density. The above nonconventional behavior is a result of the non-conventional nature of the "free-space" medium, as per the UEG theory, which is no longer a fixed but is a "flexible" or variable medium with a non-linear behavior. The electric 


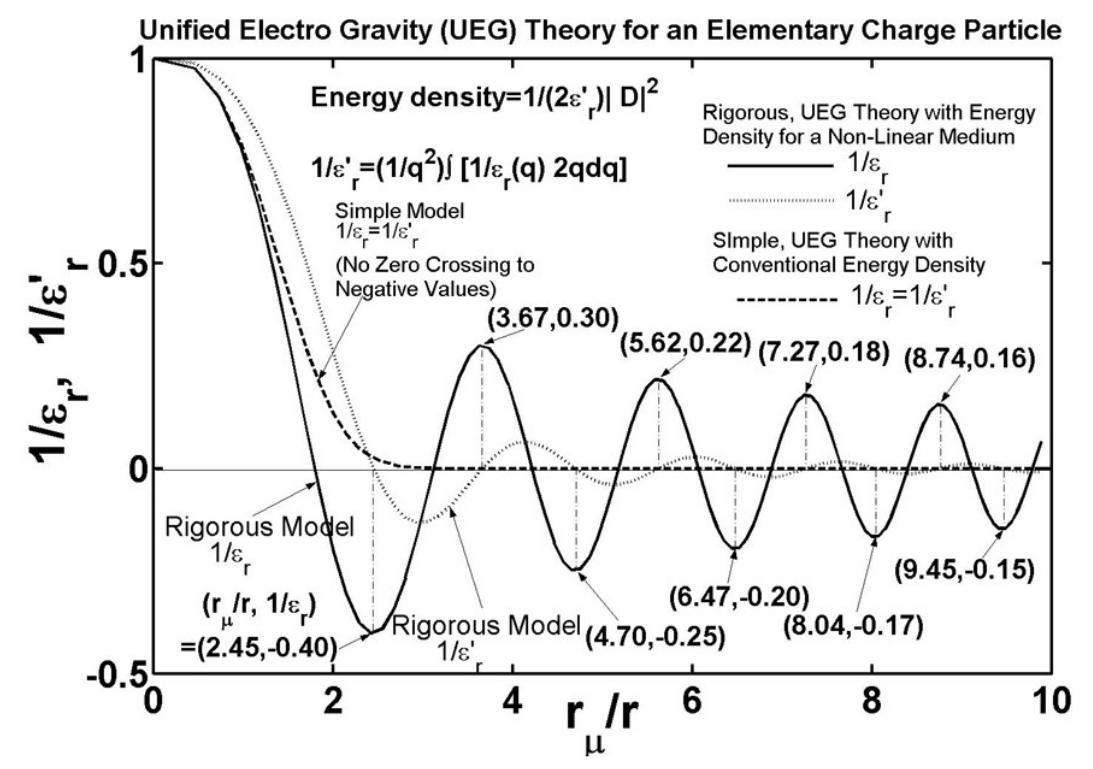

FIG. 3.

field in such a flexible medium would be a non-linear function of the source charge, and the equivalent permittivity is a function of the source charge and location. The energy density in such a non-linear medium needs to be properly re-defined as in (8), resulting in the effective inverse relative-permittivity $\underline{\epsilon}_{r}^{\prime}$ of 23 , which leads to the non-conventional disconnect between the energy density and the electric field, discussed above.

Further, the relativity permittivity $\epsilon_{r}$ from 22 in Fig 3 is allowed to be negative, which may be theoretically associated with a negative speed of light. The effective permittivity $\epsilon_{r}^{\prime}$ from 23 in Fig 3 is also allowed to be negative, which as per its definition in (8) would allow the energy density to be negative. These possibilities of negative light speed and negative energy density are remarkable new developments, not encountered in conventional physical problems, which may carry far-reaching physical and philosophical implications.

\section{B. Particle Energy and Mass, as a Function of the Charge Radius}

Once the inverse-relative permittivity function $\underline{\epsilon}_{r}(r)$ is solved, the energy density can be expressed in terms of the $\underline{\epsilon}_{r}(r)$ using (8), which can then be integrated over the total volume outside the charge radius (there is no field inside the charge radius) to obtain the total energy or the equivalent mass $m\left(=m_{0}\right.$ in $\left.(13)\right)$ of the particle.

$$
\begin{gathered}
W=\iiint_{\tau} W_{\tau} d \tau=m_{0} c^{2} \\
=\iiint_{\tau}\left[\frac{1}{16 \pi^{2} r^{4} \epsilon_{0}} \int_{0}^{q} q \underline{\epsilon}_{r}(q, r) d q\right] d \tau, \\
m=m_{0}=\frac{W}{c^{2}}=\frac{1}{4 \pi c^{2} \epsilon_{0}} \int_{r}^{\infty} \frac{1}{r^{2}} \int_{0}^{q} q \underline{\epsilon}_{r}(q, r) d q d r \\
=m_{\mu} \sum_{k=0}^{\infty} \frac{(-1)^{k} t^{\left(2 k+\frac{2}{3}\right)}}{2^{2 k}(k !)^{2}(k+1)(3 k+1)}, t=\left(\frac{r \mu}{r}\right)^{1.5}, \\
m_{\mu}=\frac{q^{2}}{8 \pi c^{2} \epsilon_{0} r \mu}=2.49 \times 10^{-30} \gamma^{-1 / 3}, \\
r_{\mu}=\left(\frac{\gamma q^{2}}{24 \pi^{2} c^{2} \epsilon_{0}}\right)^{1 / 3}=5.14 \times 10^{-16} \gamma^{1 / 3} .
\end{gathered}
$$

The charge radius in 25 is maintained as a general variable $(=r)$. The general mass function $m(r)$ in 25 would also represent the equivalent energy $\left(=c^{2} m(r)\right)$ contained in the field external to a sphere of radius $r$, produced due to the charge placed at any radius less than $r$.

Fig 4 and Fig 5 (with different mass scales/resolutions) plot the normalized mass $m / m_{\mu}$ of (25) as a function of the normalized radius $r_{\mu} / r$, showing the oscillatory behavior of the mass function, as we anticipated earlier. Any of the minimum points of the mass function would correspond to a possible stable particle with the particular charge radius, as we also anticipated. The mass $m=m_{0}$ that would have resulted, if the inverse-relative permittivity function of (24) were used in the derivation of 258, based on a simplistic (incorrect) UEG model assuming a linear medium, is expressed in 26. This 


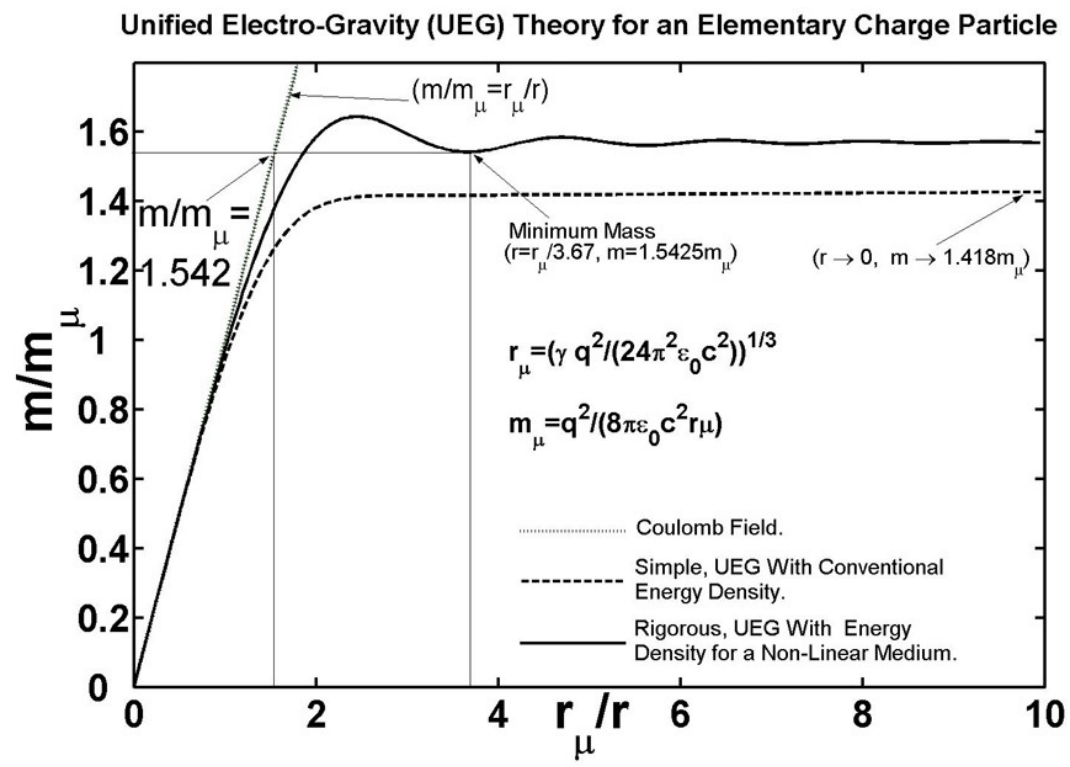

FIG. 4.

mass (26) normalized with respect to $m_{\mu}$ is also plotted in Figs 45 for reference, showing no stable radius. Also plotted in Figs 45 for reference is the normalized mass $\left(m / m_{\mu}\right)=\left(r_{\mu} / r\right)$, based on a simple Coulomb's field, which asymptotically approaches the normalized masses of (25) and (26) for $r \rightarrow \infty$, as should be expected. Clearly, the Coulomb mass does not allow any stable radius.

$$
\begin{aligned}
& m= m_{0}=\frac{W}{c^{2}}=\frac{q^{2}}{8 \pi c^{2} \epsilon_{0}} \int_{r}^{\infty} \frac{\underline{\epsilon}_{r}(r)}{r^{2}} d r \\
&=m_{\mu} \sum_{k=0}^{\infty} \frac{(-1)^{k} t^{\left(2 k+\frac{2}{3}\right)}}{2^{2 k} k !(3 k+1)}
\end{aligned}
$$

The smallest possible stable mass deduced from the oscillatory mass of 25) (Figs 45 is expected to be the mass of an electron (or a positron) without any spin. This is referred to as the static UEG mass $m_{e}^{\prime}$ of an electron. We will assume that the static UEG mass $m_{e}^{\prime}$ of an electron is about half of the total electron mass $m_{e}$, that includes additional mass/energy due to the electron's spin. This factor of about 2 between the $m_{e}^{\prime}$ and $m_{e}$ is suggested by recognizing that the electron's spin $g$-factor, as defined below in (27), is approximately equal to 2 . The bare static UEG mass $m_{e}^{\prime}$ of an electron spins effectively at the same speed and at the same radial distance as the electron's charge $q$. This would result in having the ratio of the spin magnetic moment $M$ and the spin angular momentum $p$ equal to $q /\left(2 m_{e}^{\prime}\right)$. This is equivalent to having a total electron mass $m_{e}=g m_{e}^{\prime} \simeq 2 m_{e}^{\prime}$ spinning at about half of a given speed or about half of a given radius (or at about half of a given speed-radius product), in order to produce the same given angular momentum $p$. This factor of about 2 is represented by the electron's spin $g$-factor.

$$
\begin{gathered}
\frac{M}{p}=\frac{q}{2 m_{e}^{\prime}}=\frac{g q}{2 m_{e}}, \\
g \simeq 2, \quad m_{e}^{\prime}=\frac{m_{e}}{g} \simeq \frac{m_{e}}{2} .
\end{gathered}
$$

The same conclusion may also be suggested by observing that the orbital magnetic moment of an atomic electron with an orbital angular momentum $\hbar$ is approximately equal to the magnetic moment of a spinning electron with spin angular momentum $\hbar / 2$. The approximately same magnetic moments in the two cases means the velocity-radius product of the orbital and the spinning electrons are about the same. With about the same speed-radius product, having the spin angular moment $(=\hbar / 2)$ half of the orbital angular moment $(=\hbar)$ suggests that the bare UEG static mass $m_{e}^{\prime}$ of the spinning electron is about half of the total mass $m_{e}=9.109 \times 10^{-31} \mathrm{~kg}$ of the orbiting electron.

$$
\begin{gathered}
m_{e}^{\prime} \times(v r)_{\text {spin }}=\frac{\hbar}{2}, m_{e} \times(v r)_{\text {orbital }}=\hbar \\
(v r)_{\text {spin }}=\frac{g}{2} \times(v r)_{\text {orbital }} \simeq(v r)_{\text {orbital }} \\
m_{e}^{\prime}=\frac{m_{e}}{g} \simeq \frac{m_{e}}{2}
\end{gathered}
$$

With the assumption of $m_{e}^{\prime}=m_{e} / 2$ for the minimum stable mass in Figs 45 , the value of the normalization constant $m_{\mu}$ can be calculated, from which the value of the UEG constant $\gamma$ is estimated. 


$$
\begin{gathered}
\frac{m_{e}^{\prime}}{m_{\mu}}=\frac{m_{e}}{2 m_{\mu}}=1.5425, \\
m_{\mu}=\frac{m_{e}}{3.085}=2.49 \times 10^{-30} \gamma^{-1 / 3}, \\
\gamma^{1 / 3}=3.085 \times 2.49 \times 10^{-30} / m_{e}, \\
\gamma=5.997 \times 10^{2}\left(\mathrm{~m} / \mathrm{s}^{2}\right) /\left(\mathrm{J} / \mathrm{m}^{3}\right) .
\end{gathered}
$$

As per the UEG theory of the electron, the constant $\gamma$ is declared to be a new natural constant, which is equal to a new gravitational acceleration in $\mathrm{m} / \mathrm{s}^{2}$ toward the center of gravity, produced due to one $\mathrm{J} / \mathrm{m}^{3}$ of energy density.

\section{General Relationship Between the UEG} Constant $\gamma$, the Particle Mass and Classical Radius.

The above estimate of the value of the UEG constant requires the actual UEG static mass $m_{e}^{\prime}$ of the electron. However, a general relationship between the smallest stable UEG static mass $m_{e}^{\prime}$ of an elementary particle, the corresponding classical radius $r_{e}^{\prime}$, and the UEG constant $\gamma$ required to produce the mass $m_{e}^{\prime}$, can be derived based on the expressions for the reference mass $m_{\mu}$ (25) and reference radius $r_{\mu}$ (15) used in the above analysis.

$$
\begin{gathered}
\left(\frac{m_{\mu}}{m_{e}^{\prime}}\right)^{3}=\frac{3 q^{4}}{64 \pi c^{4} \epsilon_{0}^{2} \gamma m_{e}^{\prime 3}}=\frac{3 r_{e}^{\prime 2} \pi}{\gamma m_{e}^{\prime}}, \\
\frac{\gamma m_{e}^{\prime}}{r_{e}^{\prime 2}}=3 \pi\left(\frac{m_{e}^{\prime}}{m_{\mu}}\right)^{3}, \quad m_{e}^{\prime}=\frac{q^{2}}{8 \pi \epsilon_{0} r_{e}^{\prime} c^{2}} .
\end{gathered}
$$

The value of the ratio $m / m_{\mu}=1.5425$ from the Figs 45 . for the smallest possible stable mass $m=m_{e}^{\prime}$. Using this value, the $\gamma, m_{e}^{\prime}$ and $r_{e}^{\prime}$ maybe related in term of a dimensionless constant.

$$
\frac{\gamma m_{e}^{\prime}}{r_{e}^{\prime 2}}=3 \pi\left(\frac{m_{e}^{\prime}}{m_{\mu}}\right)^{3}=34.590 .
$$

If we simply assume the total mass $m_{e}$ of the elementary particle with spin to be twice the UEG mass $m_{e}^{\prime}$, and the classical radius $r_{e}$ associated with $m_{e}$ half of that $\left(=r_{e}^{\prime}\right)$ with $m_{e}^{\prime}$, the $\gamma, m_{e}$ and $r_{e}$ may be related using a new dimensionless constant, which would be eight times the above constant.

$$
\frac{\gamma m_{e}}{r_{e}^{2}}=24 \pi\left(\frac{m_{e}^{\prime}}{m \mu}\right)^{3}=8 \times 34.590=276.720 .
$$

Notice that the above constant is close to twice the inverse-fine structure constant $1 / \alpha=137.036$, and the earlier constant in (31) is one fourth of the $1 / \alpha$, with less than one percent of difference. It may be possible that the normalized stable mass in Figs 45 is not accurate. This may reflect possible inaccuracy in computation due to poor convergence of the power series in 25, when the normalized parameter $t$ is sufficiently greater than unity ( $t$ is close to 4 at the smallest stable mass of Figs 45 ). More significantly, the small difference may also be due to lack of generality or rigor of the basic UEG static theory for the particle, presented in this paper with assumption of a simple UEG function in (11), and without including the particle's spin. The small difference may perhaps be related to the small difference between the actual value of the $g$-factor and its ideal value of 2 suggested in (27). This may point to possible physical origin of the $g$-factor associated with the spin, governed by a more rigorous version of the new UEG theory.

Leaving aside any small computational inaccuracy, or any small difference due to lack of generality or rigor of the basic UEG model, the close relations of the above dimensionless constant (31 or 32) to the fine-structure constant is intriguing. First, the very existence of a dimensionless constant based on the UEG theory, and its intriguing close numerological relationship with the known fine-structure constant $\alpha$, may strongly suggest certain fundamental basis and significance of the new UEG theory. The close numerological relationship may also strongly suggest an explicit close relationship between the UEG constant $\gamma$ associated with the dimensionless constant 31 or 32 from the UEG theory, and the particle's quantum-theoretical spin angular momentum $\hbar / 2$ (consequently, the Plank's constant $\hbar$ ) associated with the fine-structure constant $\alpha$. However, any modeling of a physically spinning particle is beyond the scope of the present UEG theory, which is valid only for a static charge. A more advanced modeling, extending the static UEG theory to model an electrodynamic problem of a physically spinning charge, would be needed in order to study any direct physical relationship between the UEG theory and the quantum spin theory (and quantum theory in general), and consequently between the associated dimensionless constant (31) or 32 and the fine-structure constant $\alpha$, respectively.

\section{THE UEG THEORY APPLIED TO MODEL THE CASIMIR EFFECT}

Consider two conducting plates placed in parallel with each other with a distance $d$, as shown in Fig.6. There would be non-zero electric fields in the external region of any conducting body, produced by all the electric charges that are naturally present in the material structure of the conductor. We are particularly interested in such fields, produced by any one of the conductors in the parallelplate structure of Fig, 6 , as observed between the two plates. These fields escape through any non-zero "gaps" between the spherical free-electrons (or, equivalently, due to the non-uniformity of the electronic arrangement) that are distributed on the conductor's surface (see Fig 6). On an average, only one spherically-shaped electron, with its static (without spin) classical radius $r_{e}^{\prime}$, would be contained in each square unit cell $r_{e}^{\prime} \times r_{e}^{\prime}$, assuming a reason- 


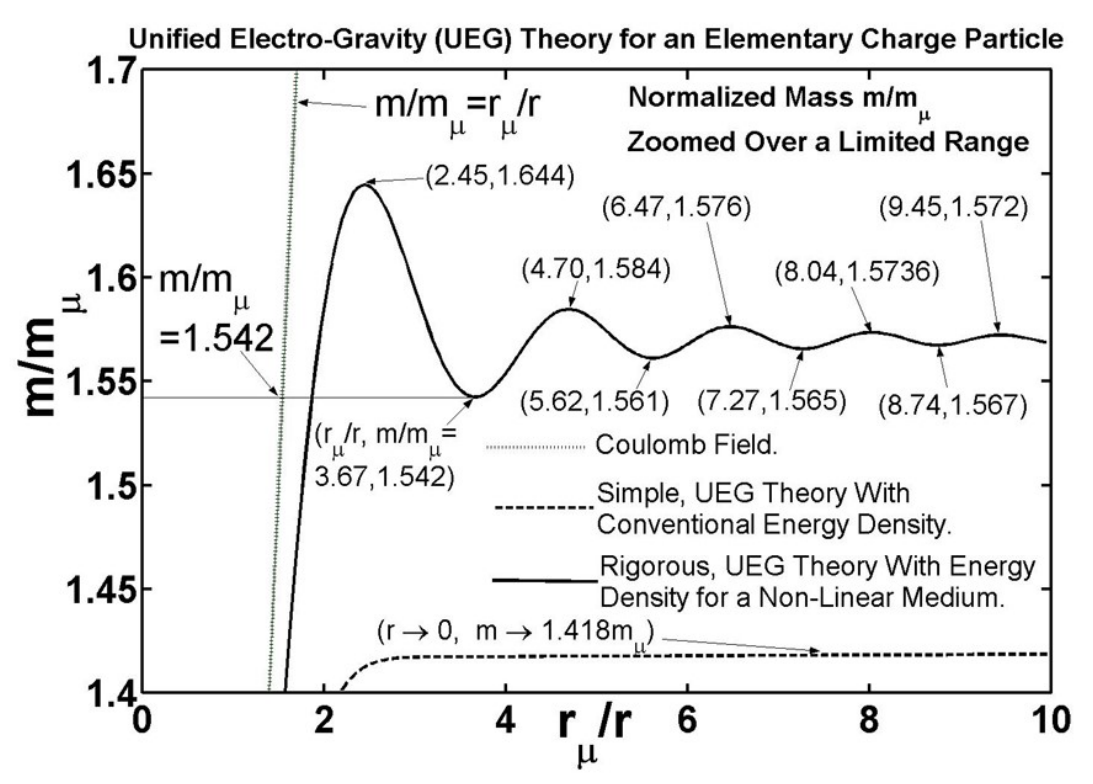

FIG. 5.

able $50 \%$ radial overlap between each pair of neighboring electrons. This topological arrangement, where the electrons with naturally circular cross-sections fill the conducting surface in a pattern of square unit cells, result in a spatially discontinuous or non-uniform distribution of the electronic charges on the conductor's surface, in contrast to a uniformly continuous distribution ideally assumed in a macroscopic model of the conductor. Therefore, the negative charges of the electrons on the surface would not completely shield or cancel the electric fields due to the equal number of positive charges bound to the atoms enclosed inside the conductor's surface, resulting in a non-zero external field.

By applying Gauss's Law of electricity to the above problem, having the equal number of the positive and the negative charges, the external electric field $E$ can be shown to be zero when integrated and averaged over a large surface, at any given time, or equivalently over a large time period at any given location. Accordingly, positive and negative fields of a given magnitude would be equally likely, canceling with each other to result in the zero average field. However, the energy density associated with a field, which is proportional to the square of the field, would be positive both for the positive as well as the negative fields. Therefore, it would result in a non-zero value for the average of the energy density, $<E^{2}>$, associated with the above zero average field. The zero average field and the non-zero average energy density $<\omega_{e}>$, produced by a conducting plate at a normal distance $d$, maybe modeled equivalent to that produced by a time-varying point charge $Q$ at the same radial distance, with the charge's average value fixed to be zero but its non-zero mean-square value $\left\langle Q^{2}>\right.$ is equal to $q^{2} / 2$, where $q$ is the elementary electronic charge.

$$
\begin{gathered}
<\omega_{e}>_{s= \pm 1 / 2}=\frac{\epsilon_{0}}{2}<E^{2}>_{s= \pm 1 / 2}=\frac{\epsilon_{0}}{2} \frac{\left\langle Q^{2}\right\rangle_{s= \pm 1 / 2}}{\left(4 \pi \epsilon_{0} d^{2}\right)^{2}} \\
=\frac{q^{2}}{64 \pi^{2} \epsilon_{0} d^{4}},\left\langle Q^{2}>_{s= \pm 1 / 2}=\frac{q^{2}}{2}\right.
\end{gathered}
$$

The field model discussed above is valid for each spin state of the electron $s= \pm 1 / 2$, that are statistically independent of each other, resulting in the average of the total energy density to be twice that of the individual energy density of each state. On the other hand, the opposing external magnetic fields, produced due to the opposing spin states $s=+1 / 2$ and $s=-1 / 2$ of the electron, statistically cancel with each other, resulting in zero total external magnetic field, and zero associated energy density. Therefore, the average of the total energy density in the external region, including both the electric and magnetic fields, is equal to the average $\left(=<\omega_{e}>\right)$ of the energy density due to only the electric field.

Now, the UEG effect due to this energy density would be responsible for an attractive gravitational field, having an acceleration $E_{g u}$ proportional to the average energy density $\left\langle\omega_{e}>\right.$, with $\gamma$ as the constant proportionality. The same UEG model was also used for modeling of a stable electron structure, as presented earlier in the paper. This UEG effect would be much stronger than the conventional (Newtonian) gravitational field due to the mass of the conducting plate, resulting in the total gravitational acceleration $E_{g}$ essentially equal to the $E_{g u}$. 


$$
\begin{aligned}
<\omega_{e}> & \left.\left.\left.=<\omega_{e}\right\rangle_{s=+1 / 2}+<\omega_{e}\right\rangle_{s=-1 / 2}=2<\omega_{e}\right\rangle_{s= \pm 1 / 2} \\
& =\frac{q^{2}}{32 \pi^{2} \epsilon_{0} d^{4}}, E_{g}=E_{g u}=\gamma<\omega_{e}>=\frac{\gamma q^{2}}{32 \pi^{2} \epsilon_{0} d^{4}} .
\end{aligned}
$$

The above UEG acceleration $E_{g}=E_{g} u$ would produce an average force $\left\langle F_{0}\right\rangle$ on each unit cell of the other conducting plate (target plate), placed parallel to the first plate which is the source of the UEG field. The $\left.<F_{0}\right\rangle_{s= \pm 1 / 2}$, experienced by each spin state $s= \pm 1 / 2$ of an electron that occupies an unit cell in the target plate, can be calculated by multiplying the mass $m_{e}$ of an electron that occupies each unit cell to the UEG acceleration $E_{g}$ calculated above. The total force $\left\langle F_{0}\right\rangle$ per unit cell would be twice the force experienced by each spin state. Note that only the free electrons on the surface of the target conductor experience the UEG force. The rest of the material body of the target plate, that are contained enclosed by its outer surface, do not experience the UEG effect, because the total electromagnetic field inside this region is assumed to be ideally zero. Now, the total UEG force $F_{u}$ on a given area $A$ of the conducting plate can be calculated by multiplying $\left\langle F_{0}\right\rangle$ by the factor $A / A_{0}$, where $A_{0}=r_{e}^{\prime 2}=4 r_{e}^{2}$ is the area of the unit cell. The $r_{e}=r_{e}^{\prime} / 2$ is the classical radius of a spinning electron, which is half of the classical radius $r_{e}^{\prime}$ of a static electron without spin, because the spinning electron is assumed to carry twice the mass of a static electron.

$$
\begin{gathered}
<F_{0}>_{s= \pm 1 / 2}=E_{g} m_{e}=\frac{\gamma q^{2} m_{e}}{32 \pi^{2} \epsilon_{0} d^{4}} \\
<F_{0}>=<F_{0}>_{s=+1 / 2}+<F_{0}>_{s=-1 / 2}=2<F_{0}>_{s= \pm 1 / 2} \\
=\frac{\gamma q^{2} m_{e}}{16 \pi^{2} \epsilon_{0} d^{4}}, F_{u}=<F_{0}>\frac{A}{A_{0}}=\frac{\gamma q^{2} m_{e} A}{64 \pi^{2} \epsilon_{0} d^{4} r_{e}^{2}}, \\
A_{0}=r_{e}^{\prime 2}=4 r_{e}^{2}, r_{e}^{\prime}=2 r_{e} .
\end{gathered}
$$

The UEG force $F_{u}$ as calculated above maybe recognized to be the Casimir force $F_{c}$ [25, 27], having the same $1 / d^{4}$ functional dependence. Equating the expressions of the two forces $F_{u}=F_{c}$, we can relate the value of the dimensionless UEG constant $\gamma m_{e} / r_{e}^{2}$ to the fine structure constant $\alpha$.

$$
\begin{gathered}
F_{u}=\frac{\gamma q^{2} m_{e} A}{64 \pi^{2} \epsilon_{0} d^{4} r_{e}^{2}}=F_{c}=\frac{\hbar c \pi^{2} A}{240 d^{4}} \\
\frac{\gamma m_{e}}{r_{e}^{2}}=\left(\frac{\pi^{3}}{15}\right)\left(\frac{4 \hbar c \pi \epsilon_{0}}{q^{2}}\right)=\frac{2.067}{\alpha} \simeq \frac{2}{\alpha}, \alpha=\frac{4 \hbar c \pi \epsilon_{0}}{q^{2}}
\end{gathered}
$$

It is seen that the UEG constant $\gamma m_{e} / r_{e}^{2}$, as estimated through the Casimir effect, is very close to the factor $2 / \alpha$ as estimated in 32 from the UEG model of an elementary particle (electron), with less than $3.5 \%$ of difference. This close relationship strengthens the validity of the proposed UEG theory, as well as the fundamental relationship of the UEG theory to the Casimir effect,

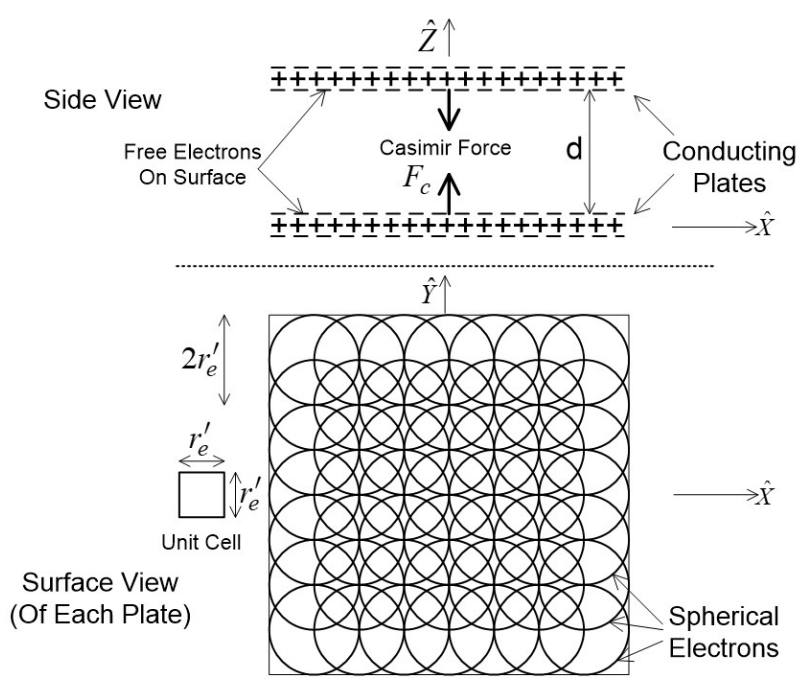

FIG. 6.

and therefore to the quantum field theory in general, on which the Casimir effect is founded upon as currently understood. This is a significant development.

\section{CONCLUSION.}

A new unified electro-gravity (UEG) theory is presented to self-consistently model a stable structure of an elementary charge particle. This is based on a nonlinear permittivity function of the empty space around the charge, which is dependent on distribution of the energy density. A new fundamental physical constant $\gamma$, referred to as the UEG constant, is introduced in order to redefine the energy density around the charge, leading to a new gravitational field which is proportional to the energy density with the $\gamma$ the constant of the proportionality. The value of the constant $\gamma$ is estimated to be about $600\left(\mathrm{~m} / \mathrm{s}^{2}\right) /\left(\mathrm{J} / \mathrm{m}^{3}\right)$, by recognizing that the lightest possible elementary charge particle is an electron (or a positron). A fundamental dimensionless constant exists, relating the mass of an elementary charge particle, its classical radius, and the UEG constant $\gamma$ required to produce the particle as the lightest possible stable particle based on the UEG theory. This dimensionless constant is shown to be closely related to the fine-structure constant $\alpha$ used in quantum electrodynamics [20, 23], with less than one percent of difference. This would strongly suggest a deeper fundamental basis of the UEG theory, which is possibly the physical origin of the fine-structure constant and related quantum-electrodynamic concepts, and could possibly be extended to model any other elementary particles.

The above possibility of a physical origin of the fine structure constant, is further strengthened by applying the same UEG theory to correctly model the functional trend of the Casimir force between two parallel conduct- 
ing plates. Equating the expression of the force between the two plates as derived from the UEG model, to that of the Casimir force as derived from a quantum-mechanical model based on zero-point vacuum energy, deduces approximately the same relationship between the UEG constant $\gamma$, mass of an electron $m_{e}$, electron's classical radius $r_{e}$, and the fine structure constant $\alpha$, as that deduced from the UEG model of an elementary particle, within just $3.5 \%$ of difference. This is a significant validation of the UEG theory, and its relation to the fine structure constant and the Casimir effect.

The energy density associated with the electric field around a charge, which is revised in this paper in terms of a new UEG function, is still not a uniquely-defined concept. The theory may be further refined and extended using higher-order UEG functions, which may explain the small differences in the different relationships between the UEG and the fine structure constants, as deduced in this paper from the particle modeling as well as the Casimir effect.

The basic UEG theory models only a static elementary charge without spin. The simple UEG theory used in this work may need to to be extended to model the elec- trodynamic problem of a spinning electron [11, which is separately pursued in 32 . The theory may be further refined and extended using higher-order UEG functions to model other elementary charge particles [3, 21, 22, such as a proton, in the standard model of particle physics [2, 33]. The basic theory for a charged particle could also be extended for neutral particles composed of concentric layers of opposite charges, and similarly for other possible composite charged or neutral particles consisting of many layers of charge particles in definite concentric patterns. Accordingly, the fundamental basis of the new UEG theory may open research avenues, providing an alternate paradigm to the existing standard model of particle physics. This could succeed in achieving the longpending unification of the electromagnetism and gravity into one complete theory, which would allow modeling of all charged and neutral particles of the standard model without need for any other additional force, possibly making the weak and strong forces currently used in the standard model redundant. Such general extensions of the basic UEG model presented in this paper, that can be applicable to all other elementary particles of the standard model of particle physics, is separately pursued in [34].
[1] J. J. Thomson, Philosophical Magazine Series 5 44, 293 (1897).

[2] N. Cottingham and D. Greenwood, An Introduction to the Standard Model of Particle Physics (2Ed) (Cambridge University Press, 2007).

[3] Wikipedia, "Leptons, Table of Leptons," http://en. wikipedia.org/wiki/Lepton, Retrieved (2013).

[4] E. Rotherford, Philosophical Magazine, Series 6 21, 669 (1911).

[5] N. Bohr, "Nobel Lecture: The Structure of the Atom," Nobel Foundation: (Retrieved August 2017) http://www.nobelprize.org/nobel_prizes/physics/ laureates/1922/bohr-lecture.html (1922).

[6] P. J. Mohr, B. N. Taylor, and D. B. Newell, Review of Modern Physics 88, 1 (2016).

[7] R. A. Millikan, Physical Review (Series I) 32, 349 (1911).

[8] G. Gabrielse and D. Hanneke, CERN Courier 46, 35 (2006).

[9] E. Schrodinger, Annalen der Physik 384, 361 (1926).

[10] R. de L. Kronig and W. G. Penney, Proceedings of the Royal Society A 130, 499 (1931).

[11] P. A. M. Dirac, Proceedings of the Royal Society A: Mathematical, Physical and Engineering Sciences 117, 610 (1928).

[12] R. P. Feynman, Physical Review 80, 440 (1950).

[13] E. J. Eichten, M. E. Peskin, and M. Peskin, Physical Review Letters 50, 811 (1983).

[14] R. P. Feynman, R. B. Leighton, and M. Sands, Lectures on Physics, Vol.II, Ch.28 (Addision Wesley, 1964).

[15] S. Ramo, J. R. Whinnery, and T. V. Duzer, Fields and Waves in Communication Electronics, 3rd Edition (John Wiley and Sons, 1993).

[16] C. A. de Coulomb, Histoire de l'Acadimie Royale des
Sciences , 569 (1785).

[17] C. A. de Coulomb, Histoire de l'Acadimie Royale des Sciences , 578 (1785).

[18] S. I. Newton, Principia: Mathematical Principles of Natural Philosophy. I. B. Cohen, A. Whitman and J. Budenz, English Translators from 1726 Original (University of California Press, 1999).

[19] S. I. Newton and S. Hawking, Principia (Running Press, 2005).

[20] A. Sommerfeld, Atomic Structure and Spectral Lines. (Translated by H. L. Brose) (Methuen, 1923).

[21] Wikipedia, "List of Baryons," http://en.wikipedia. org/wiki/List_of_baryons, Retrieved (2013).

[22] Wikipedia, "List of Mesons," http://en.wikipedia. org/wiki/List_of_mesons, Retrieved (2013).

23] R. P. Feynman, QED: The Strange Theory of Light and Matter (p. 129) (Princeton University Press, 1985).

[24] M. H. McGregor, The Power of Alpha (p. 69) (World Scientific, 2007).

[25] H. B. G. Casimir, Proceedings of the Royal Netherlands Academy of Arts and Sciences 51, 793 (1948).

[26] Wikipedia, "Casimir Effect," http://en.wikipedia. org/wiki/Casimir_effect, Retrieved (2019).

[27] R. Jaffe, Physical Review D 72, 021301 (2005).

[28] A. Einstein, Annalen der Physik 354, 769 (1916).

[29] R. F. Harrington, Time Harmonic Electromagnetic Fields (McGraw-Hill Co., 1984).

[30] J. D. Jackson, Classical Electrodynamics, 2 Ed. (John Wiley and Sons, New York, 1975).

[31] M. Abramowitz and I. Stegun (Editors), Handbook of Mathematical Functions: Bessel Functions of Integer Order (Cambridge University Press, 1964).

[32] N. Das, "Unified Electro-Gravity (UEG) Theory and 
Quantum Electrodynamics," Manuscript for Publication (2019), Available as Manuscript \#3, pp.31-42, in "A Unified Electro-Gravity (UEG) Theory of Nature," http: //wp.nyu.edu/ueg (2018).

[33] M. D. Schwartz, Quantun Field Theory and the Standard Model (Cambridge University Press, 2013).
[34] N. Das, "A Generalized Unified Electro-Gravity (UEG) Model Applicable to All Elementary Particles," Manuscript for Publication (2019), Available as Manuscript \#2, pp.14-30, in "A Unified Electro-Gravity (UEG) Theory of Nature," http://wp.nyu.edu/ueg (2018). 